\title{
Outcomes of living versus deceased donor liver transplantation: initial single center experience from Thailand
}

\author{
Worakitti Lapisatepun ${ }^{1}$, Sunhawit Jungrungsee ${ }^{1}$, Anon Chotirosniramit ${ }^{1}$, Trichak Sundhu' ${ }^{1}$, Warangkana Lapisatepun ${ }^{2}$, \\ Phuriphong Chanthima ${ }^{2}$, Settaphong Boonsri ${ }^{2}$, Suraphong Lorsomradee ${ }^{2}$, Kanya Udomsin ${ }^{1}$

\footnotetext{
${ }^{1}$ Department of Surgery, Chiang Mai university, Chiang Mai, Thailand

${ }^{2}$ Department of Anesthesiology, Chiang Mai University, Chiang Mai, Thailand
}

Background: The availability of deceased donor in the northern region of Thailand is limited. In 2015, we initiated the adult to adult living donor liver transplantation program. To date, we are the only active adult to adult living donor liver transplantation program in Thailand. We evaluated the short-term outcomes after liver transplantation compare between living and deceased donor liver transplantation.

Methods: Retrospective study of adult liver transplantation in our center since 2014. The patients were divided in to a living donor liver transplantation (LDLT) and deceased donor liver transplantation (DDLT). Baseline characteristic, perioperative data and 1-year survival were compared between two groups.

Results: There were 37 cases underwent LT in our hospital since October 2014 (20 DDLT cases and 17 LDLT cases). Baseline characteristics between two groups were not significantly difference. The operative time was longer in LDLT group $(P<0.001)$. However, cold and warm ischemic time were shorter in LDLT group ( $P<0.001$ and $P=0.026$, respectively). The median ventilator, intensive care unit and hospital stay were similar. The incidence of biliary complication was higher in LDLT group ( $47 \%$ vs $10 \%$, $\mathrm{P}<0.001)$. The vascular related complications were not significant difference. Nighty-day and 1-year survival were not significant difference between LDLT and DDLT group ( $84.6 \%$ vs. $84.2 \%$ and $76.9 \%$ vs. $84.2 \%$ respectively, $P=0.706)$.

Conclusions: In the region where deceased donor shortage, LDLT showed similar results compare to DDLT in terms of peri-operative outcomes and patient survival even done by the beginner LDLT program. However, the incidence of biliary related complication was significant higher in LDLT group.

Corresponding author: Worakitti Lapisatepun

E-mail: wlapisatepun@gmail.com

\section{(C) The Korean Society for Transplantation}

This is an Open Access article distributed under the terms of the Creative Commons Attribution Non-Commercial License (http://creativecommons.org/licenses/by-nc/4.0/) which permits unrestricted non-commercial use, distribution, and reproduction in any medium, provided the original work is properly cited. 